\title{
Desmoplastic malignant melanoma masquerading as chalazion
}

\author{
J P ROPER, T JONES, AND J D A COMMON \\ From the Department of Ophthalmology, North Staffordshire Royal Infirmary, Stoke on Trent
}

\begin{abstract}
SUMmary Desmoplastic malignant melanoma is a rare and highly malignant tumour, which usually occurs in the head and neck. This is demonstrated by a case history of a patient with this lesion on the lower lid, which has not been previously described in the British ophthalmological literature. The lesion often presents considerable problems of histological diagnosis.
\end{abstract}

A 77-year-old man was referred by his doctor with a history of a sore left lower eyelid and an area of ulceration which had been present for four months at the time of referral. He had a history of right retinal detachment operations 10 and 11 years previously. On examination his corrected visual acuities were counting fingers on the right and $6 / 18$ on the left. The intraocular tensions were raised, at $32 \mathrm{mmHg}$ on the right and $45 \mathrm{mmHg}$ on the left. The optic discs showed glaucomatous cupping. The left lower eyelid showed an area of ulceration at the junction of the medial and middle thirds, which clinically was thought to be a basal cell carcinoma.

He was prescribed $0.5 \%$ timolol maleate drops twice daily for his glaucoma, and it was planned to excise the presumed basal cell carcinoma under local anaesthetic as a day case. It was thought that the lesion could easily be excised by a one-third wedge excision and it was not photographed.

When he was admitted three weeks later, the lesion on the lid margin had extended, and a $50 \%$ excision would be necessary to achieve complete clearance with a 1-2 mm margin on either side of the lesion. One half of the left lower lid was removed by wedge excision and the lower lid reconstructed by division of the lateral canthal ligament and flap advancement. The excised specimen was sent for histological examination. The lid made a satisfactory recovery and healed well.

The initial histological diagnosis was reported as follows: 'Lower eyelid $1.5 \times 1.0 \mathrm{~cm}$, with a pale raised smooth surfaced lesion $0 \cdot 8 \times 0 \cdot 5 \mathrm{~cm}$. at the junction of the two epithelial surfaces. Microscopy. The lesion

Correspondence to Mr J D A Common, Ophthalmology Department, North Staffordshire Royal Infirmary, Stoke-on-Trent. consists of interlacing bundles of cellular fibrous tissue with interlacing bundles of cellular fibrous tissue and overlying ulceration. Degenerating eosinophil muscle fibres are present within the tumour which contains multinucleate giant cells. The appearances are of a chalazion.'

The histological diagnosis was considered incompatible with the clinical appearance of the lid, and reexamination of the slides was requested. The result was that the diagnosis was revised to that of desmoplastic malignant melanoma.

\section{PATHOLOGY}

\section{Light microscopy (Figs. 1-3)}

The specimen was fixed in neutral buffered formalin and mounted in paraffin. Routine haematoxylin and eosin stain showed an ulcerating mass situated within the epidermis, consisting of atypical spindle cells with occasional larger epithelioid cells near the surface. This extended into basal epidermis at the edge. Mitotic figures were frequent, with up to four per high field. The overlying epidermis was largely lost owing to ulceration. No pigment was present either within the neoplasm itself or in the adjacent intact epidermis. Masson's trichrome stain showed the presence of abundant collagen within the lesion; reticulin stains showed whorls of spindle cells with outlining of epithelioid cells. The Masson-Fontana stain for melanin was negative.

\section{Electron microscopy (Fig. 4)}

Pieces of neoplasm were part-fixed in osmium tetroxide and processed for electron microscopy. Sections were then examined with an AEI Corinth 500 electron microscope, which confirmed the presence of nuclear pleomorphism and abundant 


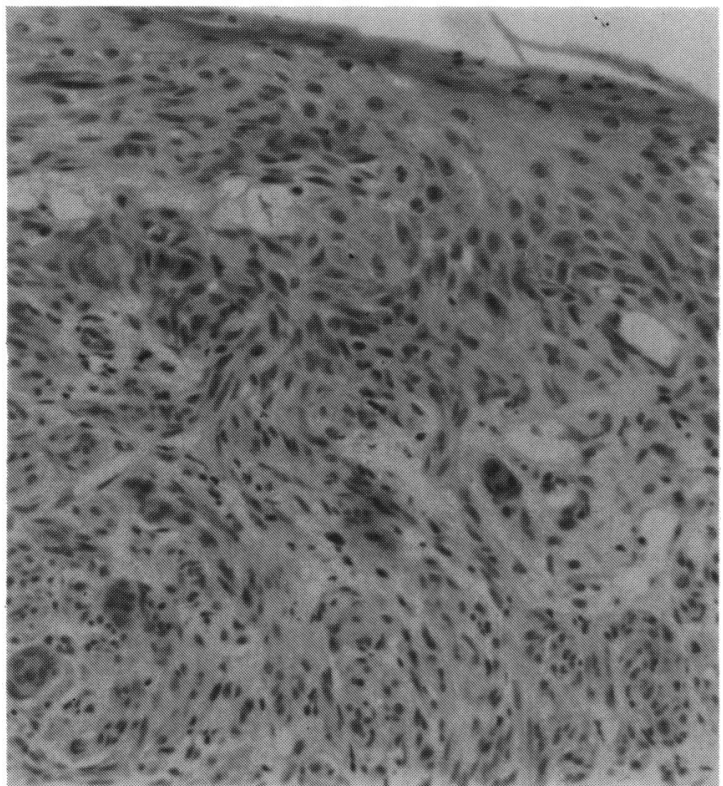

Fig. 1 Intact epidermis with underlying spindle cell elements extending into the junctional zone. Haematoxylin and eosin.

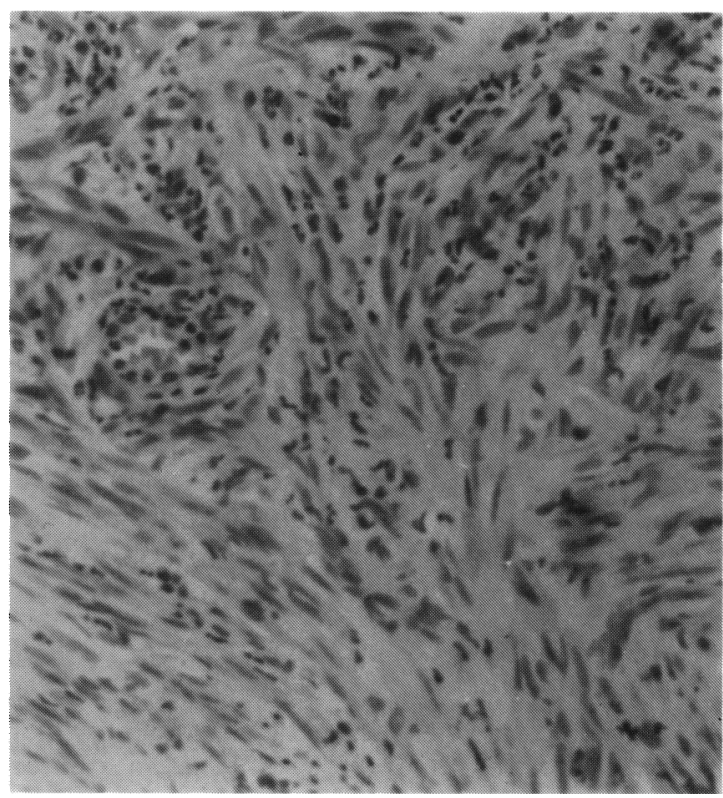

Fig. 2 Spindle cells infiltrated by polymorphs.

Haematoxylin and eosin.

Fig. 3 High power view showing frequent mitotic figures amid spindle cells with large nuclei. Haematoxylin and eosin.

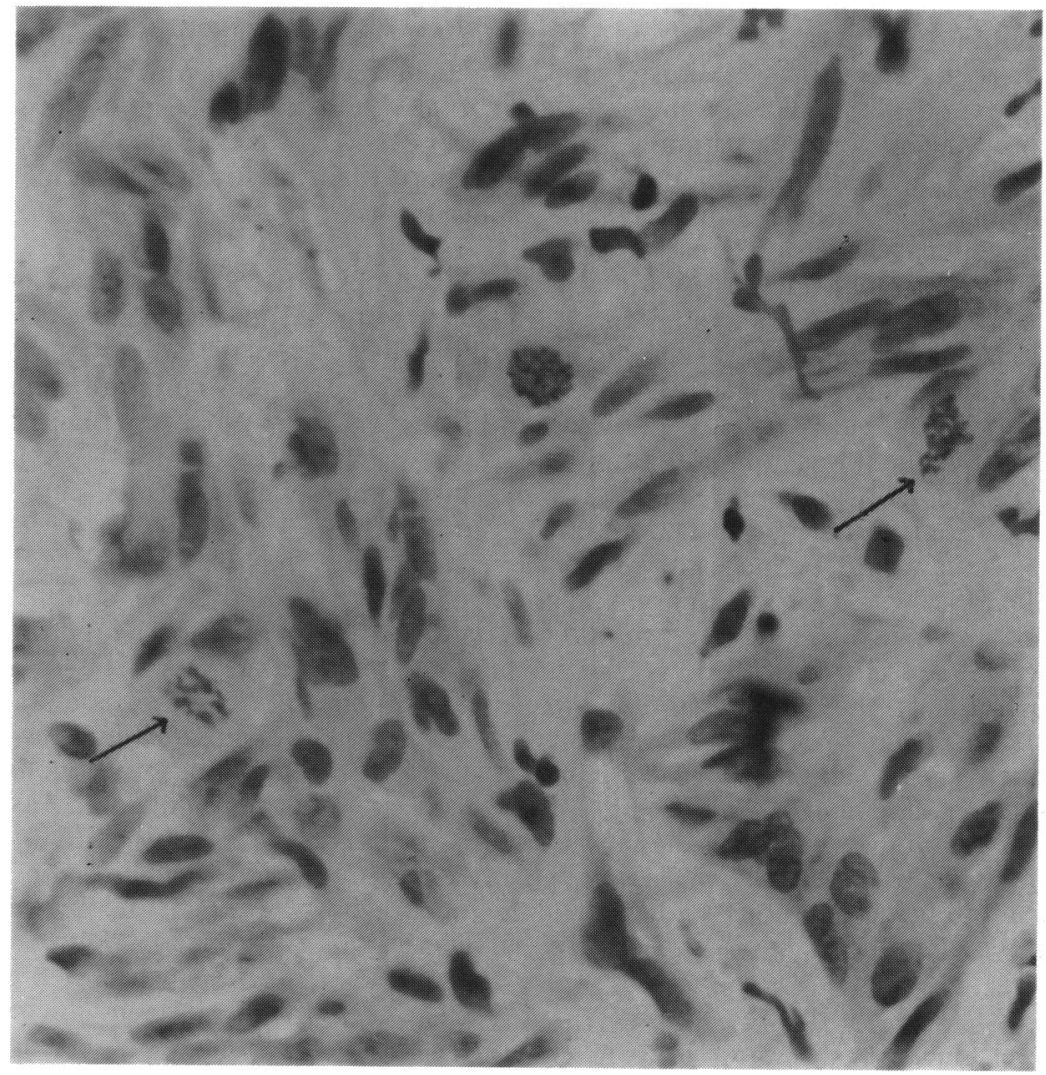




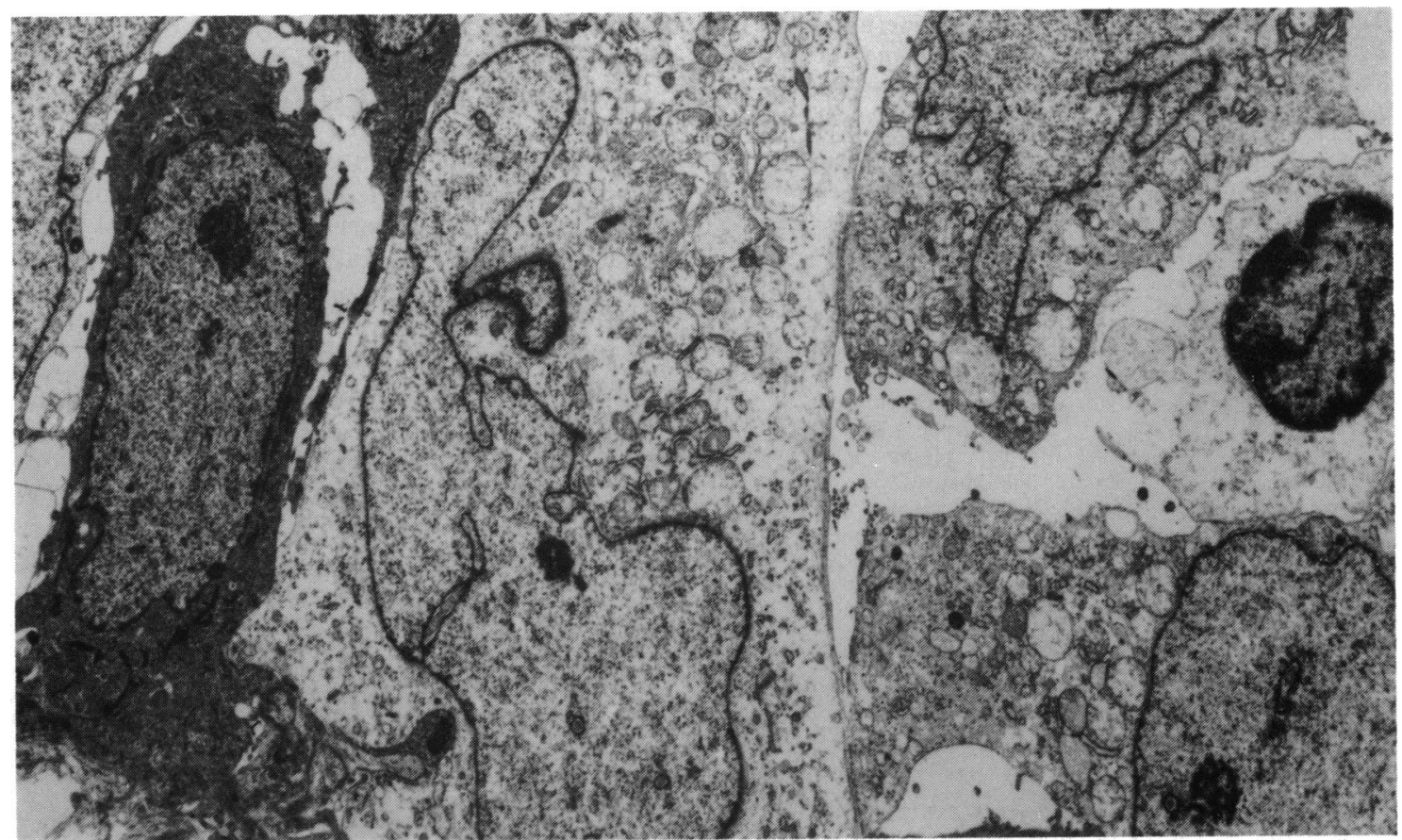

Fig. 4 Electron micrograph showing nuclear pleomorphism and swollen mitochoniria. No melanosomes are present. (×3560).

collagen. No melanosomes or premelanosomes were seen. Precollagen fibrils were not present in the cytoplasm. However, many of the cells contained dilated rough endoplasmic reticulum. The features were consistent with those of desmoplastic melanoma.

\section{Immunoperoxidase studies}

These were performed by the standard peroxidaseantiperoxidase technique on formalin-fixed, paraffinembedded tissue. Monoclonal antibody was applied against S100, a protein present in cells of neural origin, which stained the cytoplasm and nuclei of the spindle cells throughout the neoplasm, but particularly near the surface, suggesting the diagnosis of desmoplastic malignant melanoma. ${ }^{12}$

\section{Discussion}

In 1971 Conley et al. reported on seven cases in which inconspicuous, superficial, melanotic lesions resulted in bulky subcutaneous tumefactions, mainly in the head and neck. Clinically and histologically they appeared as locally invasive fibrous tumours, but the elongated neoplastic cells differed cytologically from neoplastic fibroblasts and behaved as highly malignant, recurring, and metastasising neoplasms. Some recurrences and metastases were histologically malignant melanomas. This clinicopathological entity was termed 'desmoplastic malignant melanoma. ${ }^{3}$

These patients were between 13 and 80 years of age, most being over 40 . The original red or brown lesions were flat and insignificant except one with a subcutaneous nodule. These clinically innocuous lesions were not thought to be definitely malignant initially, and in those treated by excision rather than fulguration the pathological diagnosis was very varied. Subsequently hard, subcutaneous, nodular tumours of fibrous appearance developed from six months to two years later. These recurred after excision, and metastases developed in regional lymph nodes. Visceral secondary tumours usually followed.

The total reported cases to date are 16 , nearly all from the United States, ${ }^{4}$ and the first British report was in $1982 . .^{5} 70 \%$ of cases have occurred in the head and neck. In 1982 a case of desmoplastic malignant melanoma of the upper eyelid was described. This case presented as a small pigmented lesion on the palpebral conjunctiva, which had previously been incised and curetted. Radiological examination showed demineralisation of the orbital margin. At excision there was extension of the tumour in the superior orbit. Multiple orbital recurrences followed, and two courses of radiation were ineffective. 
